QUANTUM PROBABILITY

BANACH CENTER PUBLICATIONS, VOLUME 73

INSTITUTE OF MATHEMATICS

POLISH ACADEMY OF SCIENCES

WARSZAWA 2006

\title{
FEYNMAN DIAGRAMS AND THE QUANTUM STOCHASTIC CALCULUS
}

\author{
JOHN GOUGH \\ Department of Computing $\&$ Mathematics, Nottingham-Trent University \\ Burton Street, Nottingham NG1 4BU, United Kingdom \\ E-mail: john.gough@ntu.ac.uk
}

\begin{abstract}
We present quantum stochastic calculus in terms of diagrams taking weights in the algebra of observables of some quantum system. In particular, we note the absence of non-timeconsecutive Goldstone diagrams. We review recent results in Markovian limits in these terms.
\end{abstract}

1. Introduction. Quantum stochastic calculus (QSC) involves an analysis of the fundamental quantum processes of creation/annihilation/conservation [1] and intuitively this is somehow related to emission/absorption/scattering of physical quanta as described by quantum field theory (QFT). Quantum stochastic theory has the advantages, as well as the limitations, of having a mathematically rigorous setting. It also has the theory of classical probability to fall back on for much of its inspiration. So much so that the relationship with QFT, which was originally a major motivating factor, is now frequently overlooked. Effectively, the fundamental quantum processes should be idealizations of quantum fields for some suitable "Markovian" regime. They were introduced to describe open systems dynamics: here the quantum noise couples to some quantum system and so, in some sense, we are dealing not just with traditional quantum fields, but with quantum fields taking values in the algebra of observables of some quantum system.

When presenting his famous list of problems, Hilbert is supposed to have quoted an unnamed colleague as saying that "a mathematical theory should not be considered complete until one can walk out into the street and explain it to the first person you meet". Let us suppose we did this and, as luck would have it, the first person we meet is a physicist. Would we succeed in explaining quantum stochastic calculus? My contention is that we should, though we might have to make do with some formal mathematics (presumably Hilbert wouldn't have objected?). There are many fundamental ideas, familiar to physi-

2000 Mathematics Subject Classification: Primary 81S25; Secondary 81T18.

Key words and phrases: diagrammatic techniques, quantum Markov limits.

The paper is in final form and no version of it will be published elsewhere. 
cists, hidden (sometimes too well-hidden!) in the mathematical formalism of quantum stochastic calculus. In this article, I've tried to present some basic results from quantum stochastic calculus in the language of QFT and, in particular, in terms of Feynman-type diagrams: the hope being that mathematicians and physicists will learn something from the cross-over. Diagrammatic techniques are central to QFT [2], [3], yet also of independent interest from it; they still remain an essential tool for investigating mathematical aspects of quantum theory and continue to yield some of the most illuminating insights (see, for instance, the papers [4], [5], [6]). I also want to present an account of a recent paper [7] which deals with the QSC approximation and which was originally formulated in diagrammatic language.

1.1. Expansions of evolutions. A free-particle (Bosonic) quantum field $\Phi_{t}$ living on a Hilbert (Fock) space $\mathfrak{H}$ can be decomposed into positive and negative frequency terms as $\Phi_{t}=\Phi_{t}^{(+)}+\Phi_{t}^{(-)}$: here we suppress all dependence other than time and understand the time label to refer to Heisenberg picture of the free dynamics. We take $\Phi_{t}^{(+)}$to be an annihilation field and $\Phi_{t}^{(-)}$to be a creation field. If $\Omega$ is the Fock vacuum vector, then we have the identity

$$
\Phi_{t}^{(+)} \Omega=0
$$

along with the canonical commutation relations

$$
\left[\Phi_{t}^{(+)}, \Phi_{s}^{(-)}\right]=G(t, s) .
$$

Here $G(t, s) \equiv\left\langle\Omega \mid \Phi_{t} \Phi_{s} \Omega\right\rangle$. Related to this is the propagator $K$ defined as

$$
K(t, s)=\left\langle\Omega \mid \vec{T} \Phi_{t} \Phi_{s} \Omega\right\rangle=G(t, s) \theta(t-s)+G(s, t) \theta(s-t),
$$

where $\theta(\cdot)$ is the Heaviside step function. As usual $\vec{T}$ is Dyson's chronological operation placing Heisenberg picture operators in increasing time-order from right to left.

Let $\left\{\Upsilon_{t}: t \geq 0\right\}$ be a family of self-adjoint operators on $\mathfrak{H}$ with $\Upsilon_{t}$ being some function of $\Phi_{t}^{( \pm)}$We are then interested in the evolution operator

$$
U_{t}=\vec{T}\left\{\exp -i \int_{0}^{t} \Upsilon_{s} d s\right\}
$$

by which we mean the solution to the Schrödinger equation $i \partial_{t} U_{t}=\Upsilon_{t} U_{t}, U_{0}=1$. Suppose that we have a polynomial interaction

$$
\Upsilon_{t}=\sum_{\nu} \frac{1}{\nu !} \lambda_{\nu}\left(\Phi_{t}\right)^{\nu}
$$

The standard device of quantum field theory is to expand $U_{t}$ in terms of diagrams, see, e.g. [2] or [8]. A Wick diagram $D$ is constructed as follows: choose $n=n(D)$ labelled points (vertices), each vertex will have some labelled legs attached (we let $m_{\nu}=m_{\nu}(D)$ denote the number of vertices having $\nu$ legs so that $n=\sum_{\nu} m_{\nu}$ ), we join several pairs of legs to form (undirected) edges, the result is a graph having several external lines and we now ignore the labelling. A Wick diagram is then the class of all topological equivalent 
graphs. We let $c=c(D)$ denote the number of ways we could have originally connected the various legs to get the same graph. Now denote by $\mathcal{D}_{W}$ the set of all Wick diagrams and define, for each $D \in \mathcal{D}_{W}$, the operator

$$
\hat{D}(t)=(-i)^{n} \frac{c}{n !} \prod_{\nu}\left(\frac{\lambda_{\nu}}{\nu !}\right)^{m_{\nu}} \vec{N} \int_{[0, t]^{n}} \prod_{D} K \prod_{D}\left(\Phi^{(+)}+\Phi^{(-)}\right)
$$

where $\vec{N}$ is normal ordering (placing all creation fields $\Phi^{(-)}$to the left of all annihilation fields $\left.\Phi^{(-)}\right)$and under the integral we have a factor $K\left(t_{i}, t_{j}\right)$ for each edge $(i, j)$ occurring and a factor $\Phi_{t_{k}}$ for each external line at vertex $(k)$. It is then a basic result of QFT that $U_{t}$ admits the expansion

$$
U_{t}=\sum_{D \in \mathcal{D}_{W}} \hat{D}(t)
$$

Next let $\mathcal{P}_{W}$ denote the subset of connected Wick diagrams then we may list the elements as $P_{1}, P_{2}, \cdots$ and each $D \in \mathcal{D}_{W}$ can be decomposed as $D \equiv P_{1}^{n_{1}} \times P_{2}^{n_{2}} \times \cdots$. Now one readily checks that $\hat{D}(t)=\vec{N} \frac{\hat{P}_{1}(t)^{n_{1}}}{n_{1} !} \frac{\hat{P}_{2}(t)^{n_{2}}}{n_{2} !} \cdots$ and so

$$
U_{t}=\vec{N} \sum_{n_{1}, n_{2}, \cdots} \prod_{j=0}^{\infty} \frac{\hat{P}_{j}(t)^{n_{j}}}{n_{j} !}=\vec{N} \prod_{j=0}^{\infty} \sum_{n=0}^{\infty} \frac{\hat{P}_{j}(t)^{n_{j}}}{n_{j} !}=\vec{N} \exp \sum_{P \in \mathcal{P}_{W}} \hat{P}(t) .
$$

What we have managed to do is to express the evolution operator $U_{t}$ as a normal ordered exponential of a sum over connected Wick diagrams. The connected Wick diagrams play the role of the 'primes' amongst the set of all Wick diagrams-indeed the trick of replacing a sum of products by a product of sums is just the one that goes on when we develop a prime number expansion of the Dirichlet series of a multiplicative function, the Riemann zeta function being perhaps the best known example; it is also the trick used to compute the grand canonical partition function for the free Bose gas. The result should be understood as an operator theoretic version of the usual cumulant moment expansion.

Now let $\mathfrak{h}_{S}$ be a fixed Hilbert space. We move the action up to the Hilbert space $\mathfrak{h} \otimes \mathfrak{H}$ and set

$$
\Upsilon_{t}=\sum_{\alpha, \beta} E_{\alpha \beta} \otimes\left(\Phi_{t}^{(-)}\right)^{\alpha}\left(\Phi_{t}^{(+)}\right)^{\beta}
$$

where we take $E_{\alpha \beta}^{\dagger}=E_{\beta \alpha}$. We now introduce a class of diagrams known as Goldstone diagrams - they differ from the previous ones in that the vertices are placed in time order [2]. Consider times $t_{n}>\cdots>t_{2}>t_{1}$ in the interval $[0, t]$ and draw these as vertices as shown below:

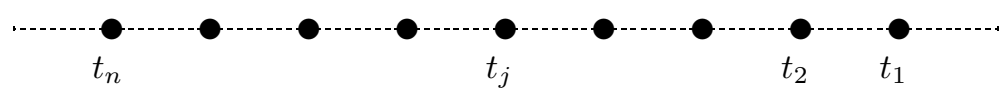

Suppose that at vertex $j$ we have $\beta_{j}$ legs coming in from the right, representing annihilators, and $\alpha_{j}$ legs going out to the left, representing creators. For example, the 
vertex for $E_{23} \otimes\left[\Phi_{t_{j}}^{(-)}\right]^{2}\left[\Phi_{t_{j}}^{(+)}\right]^{3}$ where we have $\alpha_{j}=2$ creators and $\beta_{j}=3$ annihilators is sketched as

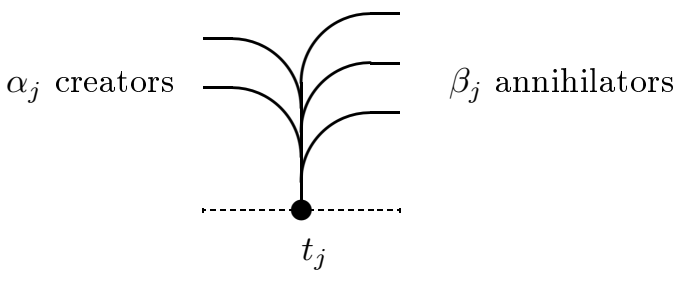

We construct a Goldstone diagram $D$ as follows. We take an arbitrary number $n=$ $n(D)$ vertices and draw in an ordered line as above. We then draw creation / annihilation lines at each vertex corresponding to one of the terms appearing in $\Upsilon$. We then connect selected creation legs to (necessarily later time) annihilation legs: the remaining uncontracted legs are then directed external lines. We consider the family $\mathcal{D}_{G}$ of all (topologically distinct) diagrams obtained in this way. To each Goldstone diagram $D$ we associate the operator

$$
\hat{D}(t)=(-i)^{n} E_{\alpha_{n} \beta_{n}} \cdots E_{\alpha_{1} \beta_{1}} \otimes \int_{\Delta_{n}(t)} \prod_{D} \Phi^{(-)} \prod_{D} G \prod_{D} \Phi^{(+)}
$$

where $\Delta_{n}(t)$ is the simplicial region $\left\{\left(t_{n}, \cdots, t_{1}\right): t>t_{n}>\cdots>t_{2}>t_{1}>0\right\}$ and we have a factor $G\left(t_{i}-t_{j}\right)$ for each edge $(i, j)$, note $t_{i}>t_{j}$, a factor $\Phi_{t_{k}}^{(+)}$for each incoming external line to a vertex $k$ and a factor $\Phi_{t_{k}}^{(-)}$for each outgoing external line. We then find the expansion

$$
U_{t}=\sum_{D \in \mathcal{D}_{G}} \hat{D}(t)
$$

In QFT one is used to switching between an expansion in terms of Goldstone diagrams (8) and one in terms of Wick diagrams (5). In the present case however we have an obstruction: the $E_{\alpha \beta}$ 's do not necessarily commute! This complication means that the Goldstone diagrams are more fundamental in the present case. The problem, of course, is that the Dyson operator $\vec{T}$ is reordering the Heisenberg fields only, while the $E_{\alpha \beta}$ 's remain in their original order.

1.2. Zero dimensional QFT. Let $a$ and $a^{\dagger}$ be annihilation operators for a single mode harmonic oscillator, We have the commutation relations $\left[a, a^{\dagger}\right]=1$ and $a \Omega=0$. Let us consider the observable $q=z a^{\dagger}+z^{*} a$ where $z$ is a complex number. The Baker-CampbellHausdorff theorem says that $\exp \{i t q\}=\exp \left\{i t z a^{\dagger}\right\} \exp \left\{-\frac{1}{2}|z|^{2} t^{2}\right\} \exp \left\{i t z^{*} a\right\}$ which here has the same content as the expansion (5). We can use a diagrammatic presentation based on two types of vertex: the creation type ....... which has weight $z$, and the annihilation type ......... which has weight $z^{*}$. If we take a vacuum expectation of $\exp \{i t q\}$ then we need only consider connected diagrams having no external lines - and there is only the one! The cumulant expansion is then

$$
\langle\Omega \mid \exp \{i t q\} \Omega\rangle=\exp \left\{\frac{(i t)^{2}}{2 !} \ldots . . . . . . . . . . .\right.
$$


and we see that $q$ is Gaussian in the vacuum state. As is well known, the odd moments vanish while the even moments are $\left\langle\Omega \mid q^{2 n} \Omega\right\rangle=|z|^{2 n} \frac{(2 n) !}{2^{n} n !}$ where the combinatorial factor counts the number of ways to partition the $2 n$ (time-ordered) vertices into $n$ (contraction) pairs. For instance, the fourth moment involves three disconnected diagrams (our convention is that we only consider the contractions as edges; the thin horizontal base line does not affect connectivity!)

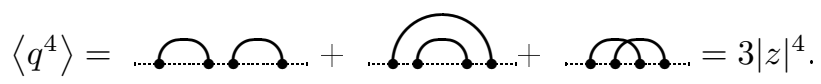

We may also consider the variable $N=(a+z)^{\dagger}(a+z)=a^{\dagger} a+z a^{\dagger}+z^{*} a+|z|^{2}$. We now introduce two extra vertices: a scattering vertex ....... with weight unity and a constant vertex ,......... with weight $|z|^{2}$. To determine the vacuum expectation of $\exp \{i t N\}$, we once again sum over all connected diagrams with no external lines. This gives

$$
\langle\Omega \mid \exp \{i t N\} \Omega\rangle=\exp \left\{\frac{(i t)}{1 !} \ldots \ldots . .+\frac{(i t)^{2}}{2 !}, \ldots \ldots+\frac{(i t)^{3}}{3 !}, \curvearrowleft \ldots+\cdots\right\}
$$

The $n^{\text {th }}$ term in the exponential will look like term has weight $|z|^{2}$ - since the scattering vertices all have weight unity. All cumulants are equal and we therefore have

$$
\langle\Omega \mid \exp \{i t N\} \Omega\rangle=\exp \left\{\sum_{n \geq 1} \frac{(i t)^{n}}{n !}|z|^{2}\right\}=\exp \left\{|z|^{2}\left(e^{i t}-1\right)\right\}
$$

and we recognize $N$ as having a Poisson distribution of intensity $|z|^{2}$. The moments of the variable $N$ are given as a polynomial of degree $n$ in $|z|^{2}$, vis.

$$
\left\langle\Omega \mid N^{n} \Omega\right\rangle=\sum_{m=1}^{n} S(n, m)|z|^{2 m}
$$

and, as is well-known in combinatorial analysis [9], the coefficients $S(n, m)$ are the Stirling numbers of the second kind: they count the number of ways to partition $n$ items into $m$ non-empty subsets. To see why they arise here, consider the following diagram contributing to $\left\langle N^{7}\right\rangle$ :

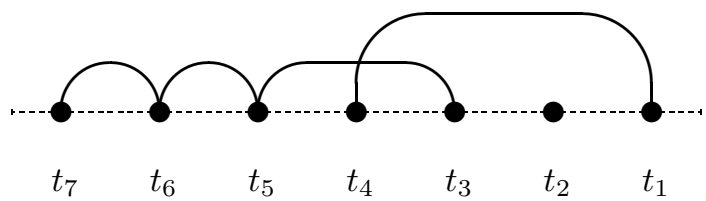

This diagram partitions the 7 vertices into 3 subsets, namely $\left\{t_{7}, t_{6}, t_{5}, t_{3}\right\},\left\{t_{4}, t_{1}\right\}$ and $\left\{t_{2}\right\}$, with each subset forming a connected sub-diagram. This contributes $\left(|z|^{2}\right)^{3}$ to $\left\langle N^{7}\right\rangle$. Consulting a textbook on combinatorics to get the Stirling numbers, we find $\left\langle N^{7}\right\rangle=\sum_{m=1}^{7} S(7, m)\left(|z|^{2}\right)^{m}=|z|^{2}+63|z|^{4}+301|z|^{6}+350|z|^{8}+140|z|^{10}+21|z|^{12}+|z|^{14}$. 
Alternatively, we could draw all $B_{7}=877$ diagrams out! The numbers $B_{n}=\sum_{m=1}^{n} S(n, m)$ counting the total number of ways to partition the $n$ vertices into non-empty subsets (of connected Goldstone diagrams) are known as the Bell numbers.

2. Quantum stochastic calculus. Remarkably, the equivalence between expansions (8) and (5) is restored in the non-commutative case in one very important situation. This is when we consider the cases $\alpha$ and $\beta$ taking only the values 0,1 in (6) and when the two-point function $G$ is replaced by a delta-function. Effectively the field is some form of quantum white noise in time. As $\alpha, \beta$ is restricted to either 0 or 1 , we shall have only four types of vertex: a constant vertex $E_{00} \otimes 1$, an emission vertex $E_{10} \otimes \Phi^{(-)}$, an absorption vertex $E_{01} \otimes \Phi^{(+)}$and a scattering vertex $E_{11} \otimes \Phi^{(-)} \Phi^{(+)}$.

The reason for the algebraic equivalence, despite the fact that the $E_{\alpha \beta}$ need not commute, is that many of the Goldstone diagrams vanish identically. This is due to the singular nature of the two-point function with respect to the simplicial integration in (7). We note that absence of certain diagrams describing moments of quantum noises has occurred elsewhere, in particular, there is an elegant description of the various forms of independent quantum processes in these terms [11].

Let us introduce some formal symbolic notations [10]. We make the replacements

$$
\Phi_{t}^{(+)} \hookrightarrow \mathfrak{a}_{t}, \Phi_{t}^{(-)} \hookrightarrow \mathfrak{a}_{t}^{\dagger}, G(t, s) \hookrightarrow \mathfrak{g}(t-s),
$$

where $\mathfrak{g}(t-s)=\kappa \mathfrak{d}_{+}(t-s)+\kappa_{-}^{*} \mathfrak{d}(t-s)$. Here $\kappa$ is a complex damping constant with $\gamma=2 \operatorname{Re}\{\kappa\}>0$. The objects $\mathfrak{d}_{ \pm}(t-s)$ are one-sided delta functions defined (for functions $f$ possessing left and right hand limits) by

$$
\int_{-\infty}^{\infty} f(s) \mathfrak{d}_{ \pm}(s-t) d s=\int_{-\infty}^{\infty} f(t+u) \mathfrak{d}_{ \pm}(u) d u=f\left(t^{ \pm}\right)
$$

Let us briefly indicate how to convert $U_{t}=\vec{T} \exp \left\{-i \int_{0}^{t} \Upsilon_{s} d s\right\}$ to normal order [10] where $\Upsilon_{t}=E_{\alpha \beta} \otimes\left(\mathfrak{a}_{t}^{\dagger}\right)^{\alpha}\left(\mathfrak{a}_{t}\right)^{\beta}$ (we use a convention from now on that repeated Greek indices are summed over values 0 and 1 ). When evaluating Goldstone diagrams, we find that if the contractions are not time-consecutive, that is, if we encounter $\mathfrak{g}\left(t_{i}-t_{j}\right)$ with $i>j+1$, then we force the multiple equalities $t_{i}=t_{i-1}=\cdots=t_{j+1}=t_{j}$ due to the time ordering, and so the contribution vanishes. Only Goldstone diagrams with time-consecutive contractions are non-zero.

Starting from the integro-differential equation $U_{t}=1-i \int_{0}^{t} \Upsilon_{s} U_{s} d s$, we have

$$
\left[\mathfrak{a}_{t}, U_{t}\right]=-i \int_{0}^{t}\left[\mathfrak{a}_{t}, \Upsilon_{s}\right] U_{s} d s=-i \int_{0}^{t} \mathfrak{g}(t-s) E_{1 \beta}\left(\mathfrak{a}_{t}\right)^{\beta} U_{s}=-i \kappa E_{1 \beta}\left(\mathfrak{a}_{t}\right)^{\beta} U_{t}
$$

or $\mathfrak{a}_{t} U_{t}=\left(1+i \kappa E_{11}\right)^{-1}\left[U_{t} \mathfrak{a}_{t}-i \kappa E_{10} U_{t}\right]$ and so

$$
\partial_{t} U_{t}=-i E_{\alpha \beta} \otimes\left(\mathfrak{a}_{t}^{\dagger}\right)^{\alpha}\left(\mathfrak{a}_{t}\right)^{\beta} U_{t} \equiv\left(\mathfrak{a}_{t}^{\dagger}\right)^{\alpha} L_{\alpha \beta} U_{t}\left(\mathfrak{a}_{t}\right)^{\beta}
$$

where

$$
L_{\alpha \beta}=-i E_{\alpha \beta}-\kappa E_{\alpha 1} \frac{1}{1+i \kappa E_{11}} E_{1 \beta} .
$$

We may interpret the conversion of the Schrödinger equation to normal ordered form as 
a change from a Stratonovich to an Itô description. This agrees with the interpretation given originally by von Waldenfels for emission-absorption interactions [12].

Having normal-ordered the Schrödinger equation, we now iterate to get

$$
\begin{aligned}
U_{t} & =1+\int_{0}^{t}\left(\mathfrak{a}_{s}^{\dagger}\right)^{\alpha} L_{\alpha \beta} U_{s}\left(\mathfrak{a}_{s}\right)^{\beta} d s \\
& =\sum_{n \geq 0} \int_{\Delta_{n}(t)}\left(\mathfrak{a}_{t_{n}}^{\dagger}\right)^{\alpha_{n}} \cdots\left(\mathfrak{a}_{t_{1}}^{\dagger}\right)^{\alpha_{1}}\left(L_{\alpha_{n} \beta_{n}} \cdots L_{\alpha_{1} \beta_{1}}\right)\left(\mathfrak{a}_{t_{1}}\right)^{\beta_{1}} \cdots\left(\mathfrak{a}_{t_{n}}\right)^{\beta_{n}} .
\end{aligned}
$$

This is reasonably familiar to quantum field theorists and such expressions can be found for instance in Berezin's book [13]. If $f$ is a suitable test function, we may consider its coherent (i.e. exponential) vector $|\varepsilon(f)\rangle$ and take $\mathfrak{a}_{t}|\varepsilon(f)\rangle=f(t)|\varepsilon(f)\rangle$ and $\langle\varepsilon(f)| \mathfrak{a}_{t}^{\dagger}=$ $\langle\varepsilon(f)| f(t)^{*}$. As (12) is normal ordered, we have no difficulty in assigning a meaning to $\left\langle\varepsilon(f) \mid U_{t} \varepsilon(g)\right\rangle$. At this stage we could just as well take (12) as the definition of the process, this is the starting point of the Maassen kernel calculus [14]. As such the time-consecutive contraction property is built into QSC, though in a way that is not readily apparent.

For the benefit of quantum probabilists, who may well be a little lost at this stage, we convert (10) into more familiar language [1]. Let $\Lambda_{t}^{\alpha \beta}=\int_{0}^{t}\left(\mathfrak{a}_{s}^{\dagger}\right)^{\alpha}\left(\mathfrak{a}_{s}\right)^{\beta} d s$ and we interpret these as the four fundamental quantum processes: $\Lambda_{t}^{00}$ is time, $\Lambda_{t}^{10}$ is creation, $\Lambda_{t}^{01}$ is annihilation and $\Lambda_{t}^{11}$ is conservation. Loosely speaking, we say $\left\{X_{t}, t \geq 0\right\}$ is adapted if $\left[\mathfrak{a}_{s}^{\sharp}, X_{t}\right]=0$ whenever $s>t$. Setting $X_{t}^{(j)}=\int_{0}^{t}\left(\mathfrak{a}_{s}^{\dagger}\right)^{\alpha} x_{\alpha \beta}^{(j)}(s)\left(\mathfrak{a}_{s}\right)^{\beta} d s$ where the $x_{\alpha \beta}^{(j)}(\cdot)$ are adapted, we see that putting to normal order yields

$$
X_{t}^{(1)} X_{t}^{(2)}=\int_{0}^{t}\left(\mathfrak{a}_{s}^{\dagger}\right)^{\alpha}\left[X_{s}^{(1)} x_{\alpha \beta}^{(2)}(s)+x_{\alpha \beta}^{(1)}(s) X_{s}^{(2)}+x_{\alpha 1}^{(1)}(s) x_{1 \beta}^{(2)}(s)\right]\left(\mathfrak{a}_{s}\right)^{\beta} d s .
$$

The basic idea goes back to Hudson and Streater [15]. In QSC, we usually write

$$
d X_{t}=x_{\alpha \beta}(t) d \Lambda_{t}^{\alpha \beta}
$$

and the above result is presented as the quantum Itô formula

$$
d\left(X^{(1)} X^{(2)}\right)=X^{(1)} d\left(X^{(2)}\right)+d\left(X^{(1)}\right) X^{(2)}+d\left(X^{(1)}\right) d\left(X^{(2)}\right)
$$

along with the quantum Itô table $d \Lambda_{t}^{\alpha 1} d \Lambda_{t}^{1 \beta}=d \Lambda_{t}^{\alpha \beta}$. The equation (10) is then interpreted as the Itô quantum stochastic differential equation $d U_{t}=L_{\alpha \beta} U_{t} d \Lambda_{t}^{\alpha \beta}$ with $U_{0}=1$. The coefficients satisfy the identities $L_{\alpha \beta}+L_{\beta \alpha}^{\dagger}+\gamma L_{1 \alpha}^{\dagger} L_{1 \beta}=0$ which are necessary and sufficient for $U_{t}$ to be an adapted, unitary quantum stochastic process. The formula for the product of several quantum integrals comes down to a normal ordering problem which can ultimately be presented as a sum over diagrams, or equivalently, a sum over partitions of the time indices: for the classical case, see [16].

3. Markov limits. Finally, we wish to comment on how regular quantum fields can approximate the singular fields considered above. Let $\lambda \neq 0$ be a parameter and consider fields $\Phi_{t}^{( \pm)}(\lambda)$ with a regular two-point function $G_{\lambda}(\cdot)$ which becomes a deltafunction in the limit $\lambda \rightarrow 0$. In particular, we may take $G(\cdot)$ to be a integrable function with $\gamma=\int_{-\infty}^{\infty} G$ and $\kappa=\int_{0}^{\infty} G$ and assume that $G(-t)=G(t)^{*}$. Then set $G_{\lambda}(t, s)=\lambda^{-2} G\left((t-s) / \lambda^{2}\right)$. We would then argue that in the limit $G_{\lambda}$ converges to 
the singular function $\mathfrak{g}$ consider above. We consider the regular unitary evolution operators

$$
U_{t}(\lambda)=\vec{T} \exp \left\{-i \int_{0}^{t} E_{\alpha \beta} \otimes\left(\Phi_{s}^{(-)}(\lambda)\right)^{\alpha}\left(\Phi_{s}^{(+)}(\lambda)\right)\right\}
$$

and we claim that for bounded $E_{\alpha \beta}$, with $\left\|\kappa E_{11}\right\|<1, U_{t}(\lambda)$ converges to the singular process $U_{t}$ considered in the last section.

The first remark that we make is that the $\lambda \rightarrow 0$ limit leads to the vanishing of each non-time-consecutive Goldstone diagram contributing to $U_{t}(\lambda)$. Moreover, when the surviving terms are computed and re-summed, we formally get the correct Itô expansion (12). Note that $L_{\alpha \beta}=-i E_{\alpha \beta}-i \sum_{r=1}^{\infty} E_{\alpha 1}\left(-i \kappa E_{11}\right)^{r-1} E_{1 \beta}$ giving the contribution to a time-consecutive block with $\alpha$ outgoing, $\beta$ incoming lines and a sum over $r$ successive scatterings in between. We see that the condition $\left\|\kappa E_{11}\right\|<1$ is necessary to sum the geometric series.

The re-summation is rather tedious, though it helps that we know what answer to expect! We also have the issue of convergence, however, we settle this below. We remark that it is sufficient to consider only the vacuum convergence as the more general situation can be inferred from this when we look at convergence in arbitrary but appropriately scaled coherent vector states.

3.1. Pulé inequalities (Gaussian). Let us start with the case where we have emission and absorption only in the interaction. The vacuum Goldstone diagrams, as we have seen in section 2, consist of $n_{2}$, say, pair contractions only. A typical diagram, one of $\frac{\left(2 n_{2}\right) !}{2^{n_{2}} n_{2} \text { ! }}$ having $2 n_{2}$ vertices, is sketched below for $n_{2}=6$ :

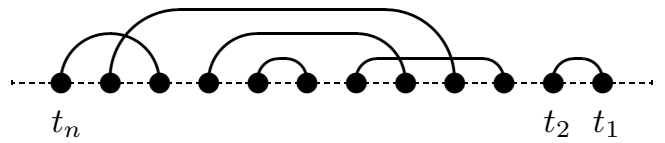

There exists a permutation $\sigma$ of the $n=2 n_{2}$ time indices which re-orders to the diagram $D_{0}(n)$ shown below

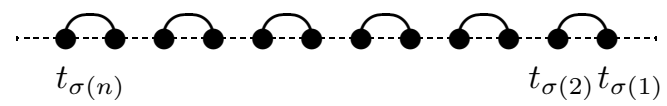

The permutation is moreover unique if it has the induced ordering of the emission times. Not all permutations on the $n$ time indices will arise this way, but the ones that do will be termed admissible. We now consider an estimate of the $n$-th term in the Dyson series:

$$
\begin{aligned}
\sum_{D \in \mathcal{D}_{G}} \int_{\Delta_{n}(t)} \prod_{D}\left|G_{\lambda}\right| & =\sum_{\text {Admissible permutations }} \int_{\Delta_{n}(t)} \prod_{D_{0}(n)}\left|G_{\lambda} \circ \sigma\right| \\
& =\int_{R(t)} \prod_{k=1}^{n_{2}}\left|G_{\lambda}\left(t_{2 k}-t_{2 k-1}\right)\right|
\end{aligned}
$$

where $R(t)$ is the union of simplices $\left\{\left(t_{n}, \cdots, t_{1}\right): t>t_{\sigma^{-1}(n)}>\cdots>t_{\sigma^{-1}(1)}>0\right\}$ over all admissible permutations $\sigma . R(t)$ will be a subset of $[0, t]^{2 n_{2}}$ and if we introduce variables $t_{2 k}$ and $s_{2 k}=t_{2 k}-t_{2 k-1}$ for $k=1, \cdots, n_{2}$ it is easily seen that the above is 
majorized by $|\kappa|^{n_{2}} \times \frac{\max (t, 1)^{n_{2}}}{n_{2} !}$. This the Pulé inequality [17] and clearly gives the uniform absolute estimate required to sum the series.

3.2. Pulé inequalities (Poissonian). We now consider scattering, and constant, terms in the interaction [7]. As we have seen there will be $B_{n}$ (the $n$-th Bell number) Goldstone diagrams contributing to the $n$-th term in the vacuum Dyson series expansion. The Bell numbers grow rapidly and have a complicated asymptotic behaviour. The proliferation of diagrams is due mainly to the multiple scattering that now may take place.

Let us consider a typical Goldstone diagram. We shall assume that within the diagram there are $n_{1}$ singleton vertices $\left[\cdots \ldots \ldots \ldots, n_{2}\right.$ contraction pairs $[\cdots \ldots \ldots \ldots \ldots], n_{3}$ contraction triples $[\cdots, \ldots \ldots \ldots]$, etc. That is the Goldstone diagram has a total of $n=\sum_{j} j n_{j}$ vertices which are partitioned into $m=\sum_{j} n_{j}$ connected subdiagrams. For instance, we might have an initial segment of a diagram looking like the following:

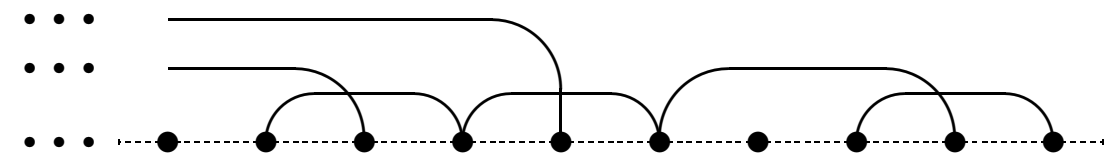

There will exist a permutation $\sigma$ of the $n$ vertices which will reorder the vertices so that we have the singletons first, then the pair contractions, then the triples, etc., so that we obtain a picture of the following type

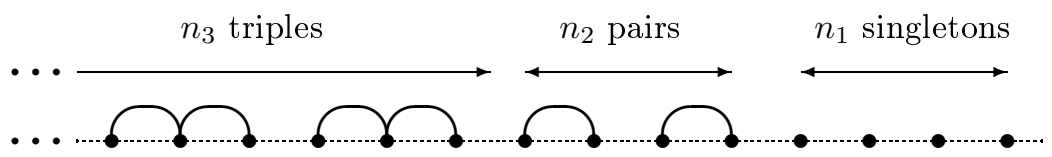

The permutation is again unique if we retain the induced ordering of the first emission times for each connected block. We now wish to find a uniform estimate for the $n$-th term in the Dyson series, we have

$$
\sum_{\text {Goldstone diagrams }} \int_{\Delta_{n}(t)} \prod\left|G_{\lambda}\right| \times \text { "weights" }
$$

where the weights are the operator norms of various products of the type $E_{\alpha_{n} \beta_{n}} \cdots E_{\alpha_{1} \beta_{1}}$. In general, the weight is bounded by

$$
\left\|E_{11}\right\|^{n_{1}+2 n_{2}+3 n_{3}+\cdots} \times C^{n_{1}+n_{2}+n_{3}+\cdots}
$$

where $C=\max _{\alpha \beta}\left\|E_{\alpha \beta}\right\|$. This is because each connected diagram of $j$ vertices will typically have one emission and one absorption, but $j-2$ scattering vertices. The Pulé argument of rearranging the sum over diagrams into a single integral over a region $R(t)$ of $[0, t]^{n}$ again applies and by similar reason we arrive at the upper bound for (13) this time of the type

$$
\sum_{n_{1}, n_{2}, n_{3}, \cdots}^{\prime}\left\|\kappa E_{11}\right\|^{n_{1}+2 n_{2}+3 n_{3}+\cdots} \times C^{n_{1}+n_{2}+n_{3}+\cdots} \times \frac{\max (t, 1)^{n_{1}+n_{2}+n_{3}+\cdots}}{n_{1} ! n_{2} ! n_{3} ! \cdots} .
$$


Here the sum is restricted so that $\sum_{j} j n_{j}=n$. A uniform estimate for the entire series is then given by removing this restriction:

$$
\Xi(A, B)=\sum_{n_{1}, n_{2}, n_{3}, \cdots} \frac{\exp \left\{\sum_{j}(A j+B) n_{j}\right\}}{n_{1} ! n_{2} ! n_{3} ! \cdots}
$$

where $e^{A}=\left\|\kappa E_{11}\right\|$ and $e^{B}=C \max (t, 1)$. Again we use the trick to convert a sum of products into a product of sums

$$
\begin{aligned}
\Xi(A, B) & =\sum_{n_{1}, n_{2}, n_{3}, \cdots} \prod_{j} \frac{\exp \left\{(A j+B) n_{j}\right\}}{n_{j} !}=\prod_{j} \sum_{n} \frac{\exp \{(A j+B) n\}}{n !} \\
& =\prod_{j} \exp \left\{e^{(A j+B)}\right\}=\exp \left\{\sum_{j} e^{A j} e^{B}\right\}=\exp \left\{\frac{e^{A+B}}{1-e^{A}}\right\} .
\end{aligned}
$$

where we need $e^{A}<1$ to sum the geometric series - this however, is precisely our condition that $\left\|\kappa E_{11}\right\|<1$.

4. Conclusions. We have established a Markov limit in the sense of [18] which we may write as

$$
\vec{T}\left\{\exp -i \int_{0}^{t} E_{\alpha \beta} \otimes\left(\Phi_{s}^{(-)}\right)^{\alpha}\left(\Phi_{s}^{(+)}\right)^{\beta} d s\right\} \hookrightarrow \vec{T}\left\{\exp -i \int_{0}^{t} E_{\alpha \beta} \otimes\left(\mathfrak{a}_{s}^{\dagger}\right)^{\alpha}\left(\mathfrak{a}_{s}\right)^{\beta} d s\right\} .
$$

On the left hand side we have a unitary which can be expanded as a normal ordered expression of the quantum fields in terms of Goldstone diagrams. The right hand side can be developed as an expansion over time-consecutive can be understood as HudsonParthasarathy unitary quantum stochastic process. We have shown the non-time-consecutive terms on the left hand side make a negligible contribution in the Markovian limit. Interpreting Weyl order as Stratonovich form and Wick order as Itô form, the above result can be considered as a non-commutative version of the Wong-Zakai limit theorem for classical processes.

The same holds for Fermi fields, however, the proof is complicated because we have to take the limit in matrix elements of appropriately scaled number states [19]. The same basic estimates suffice once more and in the limit we end up with the same process except with the $\Lambda^{\alpha \beta}$ now being Fermionic noises. As one might suspect, we have to bother ourselves collecting factors of -1 , and one would expect to obtain the same result if we dealt with $q$-commutation relations [20].

We remark that the time-ordered exponentials developed in [21] differ from the notions presented here, as we are time-ordering quantum white noises and not Itô differentials, though they do arise in models for Markov limits of discrete time systems [22].

Finally, we mention that we also have the convergence of the Heisenberg dynamics

$$
U_{t}(\lambda)^{\dagger}(X \otimes 1) U_{t}(\lambda) \text { to } U_{t}^{\dagger}(X \otimes 1) U_{t}
$$

[7]. This requires a slightly deeper analysis, however, the basic estimates above are again at the heart of things. We invite the reader to try and imagine the Goldstone diagram expansion of $U_{t}(\lambda)^{\dagger}(X \otimes 1) U_{t}(\lambda)$ to get an idea of what is involved. 
Acknowledgments. I wish to thank Professors Bożejko, Lenczewski, Młotkowski and Wysoczański and the local organisers of the 25 QP Conference in the Mathematical Research and Conference Center, Będlewo, Poland, for their most kind hospitality during which these results were presented.

\section{References}

[1] R. L. Hudson and K. R. Parthasarathy, Quantum Itô's formula and stochastic evolutions, Commun. Math. Phys. 93 (1984), 301-323.

[2] R. D. Mattuck, A Guide to Feynman Diagrams in the Many-Body Problem, McGraw-Hill, 1967.

[3] A. A. Abrikosov, L. P. Gorkov and I. E. Dzyaloshinski, Methods of Quantum field Theory in Statistical Physics, Dover Publications, New York, 1963.

[4] J. C. Baez and J. Dolan, From finite sets to Feynman diagrams, in: Mathematics Unlimited-2001 and Beyond, Engquist, Schmid (eds.), Springer, 2001.

[5] S. H. Djah, H. Gottschalk and H. Ouerdiane, Feynman graph representation of the perturbation series for general functional measures, Commun. Math. Phys., to appear.

[6] H. Yoshida, Graphical representations of the q-creation and q-annihilation operators and set partition statistics, preprint; N. Saitoh and H. Yoshida, q-deformed Poisson processess on $q$-Fock space, Journ. Math. Phys. 41 (2000), 5767-5772.

[7] J. Gough, Quantum flows as Markovian limit of emission, absorption and scattering interactions, Commun. Math. Phys. (2004).

[8] R. Ticciati, Quantum Field Theory for Mathematicians, Encyclopedia of Mathematics and its Applications 72, Cambridge University Press, 1999.

[9] J. Riordan, An Introduction to Combinatorial Analysis, New York, Wiley, 1980.

[10] J. Gough, Causal structure of quantum stochastic integrators, Theor. Math. Phys. 111 (1997), 218-233; Non-commutative Itô and Stratonovich noise and stochastic evolutions, Theor. Math. Phys. 113 (1997), 276-284; A new approach to white noise analysis, Comptes Rendus Acad. Sci. Paris 326 (1998), 981-985; The Stratonovich interpretation of quantum stochastic approximations, Potential Analysis 11 (1999), 213-233; Asymptotic stochastic transformations for non-linear quantum dynamical systems, Rep. Math. Phys. 44 (1999), 313-338; Non-commutative Markov approximations, Doklady Math. 64 (2001), 112-116.

[11] R. Lenczewski, Unification of independence in quantum probability, Inf. Dim. Anal. Quant. Probab. Rel. Topics 1 (1998), 383-405; Filtred stochastic calculus, Inf. Dim. Anal. Quant. Probab. Rel. Topics 4 (2001), 309-346.

[12] W. von Waldenfels, Stratonovich solution of the quantum stochastic differential equation describibg light emission and absorption, preprint, Universität Heidelberg, 1983; Light emission and absorption as a quantum stochastic process, preprint, Universität Heidelberg, 1982; Itô solution of the linear quantum stochastic differential equation describing light emission and absorption, in: Quantum Probability I, LNM 1055, Springer, 1986, 384-411.

[13] F. A. Berezin, Method of the Second Quantization, Academic Press, New York, 1966.

[14] J. M. Lindsay and H. Maassen, An integral kernel approach to noise, in: LNM 1303, 1988, 192-208.

[15] R. L. Hudson and R. F. Streater, Itô's formula is the chain rule with Wick ordering, Phys. Letters 86 (1982), 277-279. 
[16] G.-C. Rota and T. C. Wallstrom, Stochastic integrals: a combinatorial approach, Annals of Probability 25 (1997), 1257-1283.

[17] J. V. Pulé, The Bloch equations, Commun. Math. Phys. 38 (1974), 241-256.

[18] L. Accardi, A. Frigerio and Y. G. Lu, Weak coupling limit as a quantum functional central limit theorem, Commun. Math. Phys. 131 (1990), 537-570.

[19] J. Gough and A. Sobolev, Quantum Markovian approximations for Fermionic reservoirs, IDA QP \& Rel. Topics, to appear.

[20] M. Bożejko, B. Kümmerer and R. Speicher, q-Gaussian processes: non-commutative and classical aspects, Commun. Math. Phys. 185 (1997), 129-154.

[21] A. S. Holevo, Time-ordered exponentials in quantum stochastic calculus, in: Quantum Probability and Related Topics Vol. VII, World Sci. 1992, 175-202.

[22] S. Attal and Y. Pautrat, From repeated to continuous quantum interactions, Duke Math. J., to appear; J. Gough, Holevo ordering and the continuous time limit for open Floquet systems, Lett. Math. Phys. 67 (2004), 207-221. 